\title{
Enhancing Expression of Functional Human Sodium Iodide Symporter and Somatostatin Receptor in Recombinant Oncolytic Vaccinia Virus for In Vivo Imaging of Tumors
}

\author{
Jiahu Wang ${ }^{1}$, Rozanne Arulanandam ${ }^{1}$, Richard Wassenaar ${ }^{2}$, Theresa Falls ${ }^{1}$, Julia Petryk ${ }^{2}$, Judith Paget ${ }^{1}$, Kenneth Garson ${ }^{1}$, \\ Catia Cemeus ${ }^{1}$, Barbara C. Vanderhyden ${ }^{1,3}$, R. Glenn Wells ${ }^{2}$, John C. Bell*1,4, and Fabrice Le Boeuf*1 \\ ${ }^{I}$ Centre for Innovative Cancer Research, Ottawa Hospital Research Institute, Ottawa, Ontario, Canada; ${ }^{2}$ Cardiac PET Research, \\ University of Ottawa Heart Institute, Ottawa, Ontario, Canada; ${ }^{3}$ Department of Cellular and Molecular Medicine, University of \\ Ottawa, Ottawa, Ontario, Canada; and ${ }^{4}$ Department of Biochemistry, Microbiology and Immunology, University of Ottawa, Ottawa, \\ Ontario, Canada
}

\begin{abstract}
Oncolytic virus (OV) therapy has emerged as a novel tool in our therapeutic arsenals for fighting cancer. As a live biologic agent, OV has the ability to target and selectively amplify at the tumor sites. We have reported that a vaccinia-based OV (Pexa-Vec) has shown good efficacy in preclinical models and in clinical trials. To give an additional tool to clinicians to allow both treatment of the tumor and improved visualization of tumor margins, we developed new viral-based platforms with 2 specific gene reporters. Methods: We incorporated the human sodium iodide symporter $(h N / S)$ and the human somatostatin receptor 2 ( $h S S R 2$ ) in the vaccinia-based OV and tested viral constructs for their abilities to track and treat tumor development in vivo. Results: Early and high-level expression of $h N I S$ is detrimental to the recombinant virus, leading to the aggregation of hNIS protein and early cell death. Putting $h N I S$ under a late synthetic promoter allowed a higher functional expression of the protein and much stronger ${ }^{123}$ or ${ }^{99} \mathrm{Tc}$ uptake. In vivo, the hNIS-containing virus infected and amplified in the tumor site, showing a better efficacy than the parental virus. The hNIS expression at the tumor site allowed for the imaging of viral infection and tumor regression. Similarly, $h S S R 2$-containing OV vaccinia infected and lysed cancer cells. Conclusion: When tumor-bearing mice were given $h N I S$ - and $h S S R 2$-containing OV, ${ }^{99} \mathrm{Tc}$ and ${ }^{111} \mathrm{In}$ signals coalesced at the tumor, highlighting the power of using these viruses for tumor diagnosis and treatment.
\end{abstract}

Key Words: animal imaging; oncology; Gl; SPECT/CT; cancer imaging; oncolytic viruses; $h N I S ; h S S R 2$

J Nucl Med 2017; 58:221-227

DOI: 10.2967/jnumed.116.180463

\section{D} iagnosing and treating cancer remain big challenges for clinicians and researchers. Early tumor diagnosis and sensitive imaging of tumor locations provide valuable insight into disease progression and may facilitate determination of the appropriate therapeutic approach to maximize treatment efficacy. Recent preclinical and clinical research has demonstrated oncolytic viral

Received Jun. 29, 2016; revision accepted Aug. 4, 2016.

For correspondence or reprints contact: Fabrice Le Boeuf, Ottawa Hospital

Research Institute, 501 Smyth Rd., Ottawa, Canada K1H8L6.

E-mail: fleboeuf@ohri.ca

${ }^{*}$ Contributed equally to this work.

Published online Sep. 15, 2016.

COPYRIGHT (C 2017 by the Society of Nuclear Medicine and Molecular Imaging. therapy as a promising alternative in fighting cancer (1-4). Oncolytic viruses are commonly based on engineered viruses that are attenuated, armed, or retargeted (4,5). Poxvirus, particularly vaccinia virus (VV), has been shown to be a good candidate.

The poxvirus genome can be easily genetically modified to accommodate inserts as large as $25 \mathrm{~kb}$, using strategies that are dependent on homologous recombination (6). With the use of these approaches, poxvirus family members have proven to be valuable vectors for gene therapy in several therapeutic applications (7-17) and have shown promise as novel vaccines against such diseases as Japanese encephalitis virus (JEV) (18), malaria (19), tuberculosis (20), and cancer $(7,8,12,13)$. Oncolytic vaccinia is known to infect a wide range of cells but only productively replicates in cancer cells with a persistent viral gene expression (21-23). Exploiting the vaccinia late promoter for the expression of genes of interest may provide the opportunity to maintain expression of the transgene over several days specifically in the tumor environment where viral replication is favored. Therefore, VV could be the platform of choice to express specific genes that localize to and allow visualization of evolving tumors.

Two genes of potential interest for expression in the vaccinia platform were the sodium/iodide symporter (NIS) (24) and the somatostatin receptor type 2 (SSR2) (25). NIS expression has been extensively used for imaging in combination with radiolabeled ${ }^{99} \mathrm{Tc}$ or ${ }^{131} \mathrm{I}$. NIS has been exploited therapeutically in thyroidrelated disease, and ectopic expression of this gene has also been important to extend the use of radioiodine for nonthyroid tumors $(26,27)$. Notably, previous studies reported the use of measles virus as a platform expressing NIS and research that has led to a current phase 1 clinical trial for ovarian cancer (28). SSR2 is a member of the superfamily of somatostatin receptors whose ligand is the peptide hormone somatostatin (29). Interestingly, somatostatin has also been shown to inhibit proliferation of many solid tumors, notably pancreatic cancer using a somatostatin analog. Sandostatin or octreoide acetate has been widely used in treating carcinoid tumors (30).

Here, we describe the construction of 2 new recombinant VVs harboring $h N I S$ or $h S S R 2$ as in vivo tumor imaging candidates and as cancer therapeutic candidates. Our data indicate that high-level expression of human NIS (hNIS) and hSSR2 in recombinant VV from the early promoter leads to lower viral titers. In addition, the late promoter, although considered weaker than the early promoter, led to higher levels of hNIS expression on the membrane of 
the infected cells. Consequently, the uptake of iodide and technetium is also greatly improved using a late promoter in recombinant vaccinia vectors. Imaging or therapeutic virus candidates were found to preferentially replicate in various tumor sites in vivo and can be clearly mapped using small-animal SPECT/CT without affecting normal tissues. hNIS-encoding virus has also been shown to be effective at killing tumor cells in vivo, suggesting a possible role as a therapeutic agent. Our results strongly suggest that these novel vaccinia recombinants expressing human sodium iodide symporter (hNIS) or human somatostatin receptor 2 (hSRR2) may be useful to localize, visualize, follow, and treat various types of tumor and warrant further investigation in a clinical setting.

\section{MATERIALS AND METHODS}

\section{Antibodies and Reagents}

Primary mouse or rabbit anti-hNIS (Abcam), anti-hSSR2 (Novus Biologics), Ki-67 (DAKO), VV Quartett (Quartett), and anti- $\beta$-actin (Sigma) antibodies were used. Secondary antibodies were conjugated to horseradish peroxidase (BioRad) or to Cy5 (Abcam) for use in immunoblotting and immunofluorescence, respectively.

\section{Immunohistochemistry Staining}

Quartett Immunohistochemistry. Antivaccinia polyclonal antibody and secondary antibody kits (Vectastain; Vector Laboratories) were used for immunohistochemistry.

Ki-67 Immunohistochemistry. Paraffin-embedded sections were boiled in citrate buffer (10 mmol/L, pH 6). Primary anti-Ki-67 antibody (1:25 dilution; DAKO) was applied overnight and detected using an antirat antibody detection system (DAKO).

Cells. Human osteosarcoma (U2OS), human colorectal (HT29, HCT116), human renal cell adenocarcinoma (768-O), and human breast cancer (HeLa, MCF-7) cells were from American Type Culture Collection. Cell lines were maintained in Dulbecco modified Eagle medium (Hyclone) supplemented with 10\% fetal calf serum (Cansera), penicillin $(100 \mathrm{U} / \mathrm{mL})$, and streptomycin sulfate $(100 \mu \mathrm{g} / \mathrm{mL})$ at $37^{\circ} \mathrm{C}$ in $5 \% \mathrm{CO}_{2}$.

\section{Plasmids}

Plasmids pSC65-EGFP-p7.5- $h N I S$ and $-h S R$ were constructed based on pSEL-eGFP (gift from Dr. David L. Bartlett, Pittsburgh University) (22). Primers for late promoter sequences were annealed and ligated to form pSC65-EGFP-p00/p04-hNIS and pSC65-EGFPp00/p04-hSR.

\section{Primers for Late Promoter Construction}

Primers for late promoter construction were as follows: P00 (forward): agcttACAAAAAAAACTTCTCTAAATAGAc; P00 (reverse): tcgagTCTATTTAGAGAAGTTTTTTTTGTa; P04 (forward): agcttACAAAAAAAACTTCTCCAAATAGAc; P04 (reverse): tcgagTCTATTTGGAGAAGTTTTTTTTGTa. Recombinant viruses were constructed as previously described (6).

\section{In Vivo Animal Model}

HT29 $(3 \times 10 \mathrm{e} 6)$ tumors were established subcutaneously in 6-wk-old CD1 female nude mice $(n=5)$. Palpable tumors formed within $11 \mathrm{~d}$ after seeding. VV-eGFP or VV-hNIS was administered $(1 \times 10 \mathrm{e} 7 \mathrm{pfu} /$ mouse). For the efficacy studies, HT29 tumors were treated and measured every $2-4 \mathrm{~d}$ using an electronic caliper. Tumor volume was calculated as $\left(\mathrm{L}_{1}\right)^{2} \mathrm{xL}_{2} / 2$. For the ovarian cancer model, T-antigen- positive tgMISIIR-TAg (Tg(Amhr2-SV40TAg)1Bcv) female transgenic mice (31) were treated with weekly intraperitoneal injections of phosphate-buffered saline (PBS) or $1 \times 10^{8} \mathrm{pfu}$. control and hNIScontaining VV starting at $10 \mathrm{wk}$ of age. Mice survival times were recorded.

\section{In Vivo Green Fluorescence Protein (GFP) Detection}

VV-infected mice were anesthetized using isoforane. The bioluminescence was detected using a 200 Series IVIS Imager (Xenogen) and Living Image software (version 2.5; Xenogen).

\section{Fluorescence Microscopy}

To detect hNIS production from recombinant VV, U2OS cells $(2 \times$ $10^{5}$ ) were seeded and infected with virus (multiplicity of infection $[\mathrm{MOI}]=0.01)$ for $2 \mathrm{~d}$. The cells were washed (PBS) and fixed (4\% paraformaldehyde) for $10 \mathrm{~min}$. The cells were treated for $30 \mathrm{~min}$ with blocking buffer $(0.2 \%$ Triton X-100, 5\% goat serum) and incubated overnight at $4{ }^{\circ} \mathrm{C}(1: 100$ anti-hNIS). The cells were washed with PBS, incubated for $30 \mathrm{~min}$ (Cy3-conjugated goat anti-rabbit), washed 3 times, and mounted on a slide. DNA was stained with 4,6-diamidino2-phenylindole (DAPI; $1.5 \mu \mathrm{g} / \mathrm{mL}$ ). Cell images were captured using a Zeiss Axioplan-2 microscope equipped with Axioview 3.1 software.

\section{Virus DNA Extraction, Polymerase Chain Reaction, and Sequencing}

DNA was extracted from purified virus stocks by adding a lysis buffer (5 mM Tris, $\mathrm{pH} 8.0 ; 2.5 \%$ polysorbate-20; $2.5 \% \mathrm{NP}-40 ; 250 \mathrm{mg} / \mathrm{mL}$ proteinase K) followed by incubation at $55^{\circ} \mathrm{C}$ for $4 \mathrm{~h}$. Samples were boiled $10 \mathrm{~min}$ and DNA-purified by phenol extraction. Regions spanning the thymidine kinase gene were amplified by polymerase chain reaction and sequenced to confirm the virus harboring the intended inserts.

\section{Western Blotting}

Cells were infected, washed with PBS, and lysed with mammalian cell lysis buffer or ProteoJET membrane protein extraction buffer (Fermentas) with protease inhibitors (Complete; Roche) for $30 \mathrm{~min}$ on ice. Cell lysates were sonicated for $30 \mathrm{~s}$ and centrifuged at $10,000 \mathrm{~g}$ and cell pellets discarded, fractionated by electrophoresis on a Nupage $4 \%-$ $12 \%$ Bis-Tris gel (Invitrogen), transferred to hybond-C extra nitrocellulose membranes (Amersham), and probed with the indicated primary antibodies. Secondary antibodies conjugated to horseradish peroxidase were used to detect bound antigens.

\section{In Vitro Radioactive Isotope Uptake}

U2OS cells were seeded in 12 -well plates at $2 \times 10^{5}$ cells per well. Twenty-four hours later, cells were infected with VV at an MOI of 0.1. Two days after infection, cells were washed once with $1 \mathrm{~mL}$ of prewarmed $\left(37^{\circ} \mathrm{C}\right)$ Hank's balanced salt solution (HBSS) (Invitrogen). Cells were then incubated with $0.9 \mathrm{~mL}$ of warm (4-(2-hydroxyethyl)-1-piperazine)ethanesulfonic acid (HEPES; $10 \mathrm{mM}$ )-buffered HBSS and $0.1 \mathrm{~mL}$ of ${ }^{125} \mathrm{I}$ solution $(\sim 0.37 \mathrm{MBq}$ or $\sim 10 \mu \mathrm{Ci}$ or $\sim 100,000 \mathrm{cpm}$ ) for $45 \mathrm{~min}$ at $37^{\circ} \mathrm{C}$. As a control, a subset of samples infected with the NIS-expressing viruses were incubated with $0.8 \mathrm{~mL}$ of warm HEPES-HBSS, $0.1 \mathrm{~mL}$ of $100 \mathrm{mM}$ potassium perchlorate $\left(\mathrm{KClO}_{4}\right)$, and $0.1 \mathrm{~mL}$ of the ${ }^{125} \mathrm{I}$ solution. Cells were washed twice with $1 \mathrm{~mL}$ of ice-cold HEPES-HBSS before being lysed with $1 \mathrm{~mL}$ of $1 \mathrm{~N} \mathrm{NaOH}$ for $15 \mathrm{~min} .{ }^{125}$ I radioactivity of the cell lysates was measured by a $\gamma$-counter and presented in cpm per $1 \times 10^{5}$ cells on average over triplicates.

\section{Small-Animal SPECT Imaging}

Images were acquired on a 4-head small-animal SPECT/CT camera (NanoSPECT; Mediso). A 9-hole 1-mm-diameter multipinhole collimator was attached to each head. For all studies, a spiral CT (45 kVp, 
$500 \mathrm{mAs} /$ projection) was acquired to assist with localization of the radiotracer distributions. The expression of NIS was visualized through uptake of ${ }^{99 \mathrm{~m}} \mathrm{Tc}$-pertechnetate $\left(\mathrm{NaTcO}_{4}\right)$. Imaging was acquired with a $140 \pm 14 \mathrm{keV}$-energy window starting 30 min after a $75-$ $95 \mathrm{MBq}(2-2.5 \mathrm{mCi})$ intraperitoneal injection of pertechnetate. Images were acquired over 30-60 min. To image SSR2, $11 \mathrm{MBq}(300 \mu \mathrm{Ci})$ or $26 \mathrm{MBq}(700 \mu \mathrm{Ci})$ of ${ }^{111} \mathrm{In}$-octreotide were injected intravenously. Images were acquired for 30-60 min starting $24 \mathrm{~h}$ after injection using energy windows over the 2 photopeaks of $173 \pm 17$ and $245 \pm$ $25 \mathrm{keV}$. In the dual-isotope images, both ${ }^{99 \mathrm{~m}} \mathrm{Tc}$ and ${ }^{111} \mathrm{In}$ images were acquired simultaneously, providing exact coregistration. For the ${ }^{131}$ I therapy study, $37 \mathrm{MBq}(1 \mathrm{mCi})$ of ${ }^{131} \mathrm{I}$ were injected intraperitoneally once per week for $3 \mathrm{wk}$. A pertechnetate image was obtained before the ${ }^{131}$ I injection to evaluate the presence of NIS expression in the tumor.

\section{Animal Care}

All animals were handled in strict accordance with good animal practice as defined by the relevant national and local animal welfare bodies, and all animal work was approved by the appropriate committee (University of Ottawa, Animal Care Committee, ME-220 protocol, Dr. John Bell).

\section{RESULTS}

\section{Recombination of hNIS and hSSR2 with Vaccinia Wyeth Strain}

Wyeth VV strain with loss of the thymidine kinase gene were shown to have significantly decreased pathogenicity compared with wild-type virus and more specificity to tumor tissues. This vaccinia locus was chosen for insertion of the hNIS or hSSR2. The target insertion site for $h N I S$ and $h S S R 2$ is shown in a schematic of the shuttle vector generated for recombination into the VV-thymidine kinase locus (Figs. 1A and 2A). Because hNIS and hSSR2 recombinant viruses are intended for imaging the productive infection at tumor sites, late expression of $h N I S$ is more desirable for a stronger expression in the cells. Besides the early and late promoter p7.5, we have also expressed the $h N I S$ and the $h S S R 2$ with a synthetic late promoter p00 and p04. Selected viral plaques are depicted in Supplemental Figure 1 (supplemental materials are available at http:// jnm.snmjournals.org) and show no obvious difference in term of size compared with parental virus used to make recombinants. After a GFP flow cytometer-based selection, viruses were plaque-purified multiple times.

The expression of hNIS from recombinant viruses was determined in both infected cancer cells (HeLa) and normal cells (GM38) (Figs. 1B and 1C). First, VV-hNIS using either the p00 or the p04 late promoters shows strong tropism for tumor cells based on the enhanced expression of GFP (Fig. 1B), the higher virus titers (Fig. 1C), and the enhanced expression of hNIS (Fig. 1D) in the tumor cell line when compared with normal cells. Although specific hNIS expression was confirmed and as expected according to the literature by Western blot (Fig. 1D) $(32,33,34)$, the functionality of the symporter was validated by measuring the accumulation of ${ }^{131} \mathrm{I}$ or ${ }^{99 \mathrm{~m}} \mathrm{Tc}$ radioisotopes into the cells (Figs. $1 \mathrm{E}$ and $1 \mathrm{~F}$ ). This accumulation is NIS-specific because $\mathrm{KClO}_{4}$ can block the uptake. $h N I S$ driven by late promoters again is found to be more active than expression from the early late p7.5 promoter when cells were infected with the same MOI (Fig. 1E).

In parallel, expression of hSSR2 from recombinant VV-hSSR2 viral constructs was validated by immunofluorescence (Figs. 2B and 2C). Specific staining for SSR2 in infected cells (GFP-

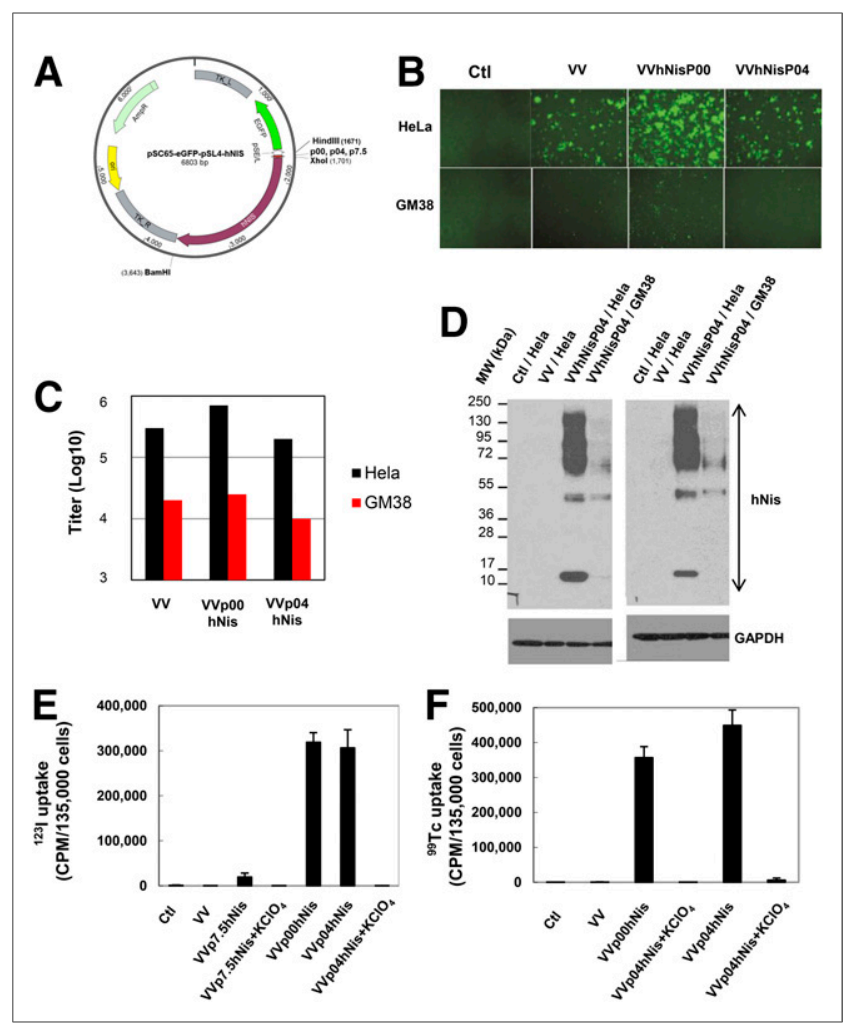

FIGURE 1. Late promoter allows high-level expression of functional hNIS using vaccinia-based platform. (A) Diagram of shuttle vector used to generate recombinant $h N I S$ VV. (B) GFP images $48 \mathrm{~h}$ after infection of HeLa and GM38 uninfected (Ctl) or infected with VV or VVNIS p00 or p04. (C) VV titers, $48 \mathrm{~h}$ after infection of HeLa and GM38. (D) Western blot for NIS expression. HeLa cells were uninfected (Ctl) or infected with VV or VV-NIS p00 or p04. Sample lysate collections were done $48 \mathrm{~h}$ after infection and screened by Western blot. $(E){ }^{131}$ I uptake of U2OS cells, uninfected (Ctl) or infected with VV or VV-hNISexpressing recombinants. Inhibition of the hNIS was achieved by treatment with $\mathrm{KCLO}_{4}$. (F) ${ }^{99} \mathrm{Tc}$ uptake of U2OS cells, uninfected (Ctl) or infected with VV or VV-hNIS-expressing recombinants. Inhibition of the hNIS symporter using $\mathrm{KCLO}_{4}$. GAPDH = glyceraldehyde 3phosphate dehydrogenase.

expressing) confirmed both expression and localization of SSR2 (Figs. 2B and 2C). Interestingly, using an early/late promoter (p7.5) or a late promoter (p04) changed the localization of SRR2. In fact, aggregation was observed with the early/late promoter, and a more homogeneous distribution suggesting a better functionality was observed using a late vaccinia promoter (Fig. 2C).

\section{Late Promoter hNIS-Encoding Virus Can Infect and Lyse Cancer Cells}

hNIS oncolytic virus candidates were tested in vitro and in vivo for their ability to spread in tumor cells and their therapeutic potential. First, we observed that hNIS virus can infect and replicate in various types of human tumor cells-colon carcinoma HT29 and HCT116, renal carcinoma 786-O, osteosarcoma U2OS, or breast tumor cells (Figs. 3A and 3C) -leading to high ectopic expression of hNIS (Fig. 3B). As expected, VV-hNIS platforms incorporating the late promoters p00 or p04 replicate in the subcutaneous human HT-29 immunocompromised mouse tumor model, as demonstrated by viral eGFP expression assessed 


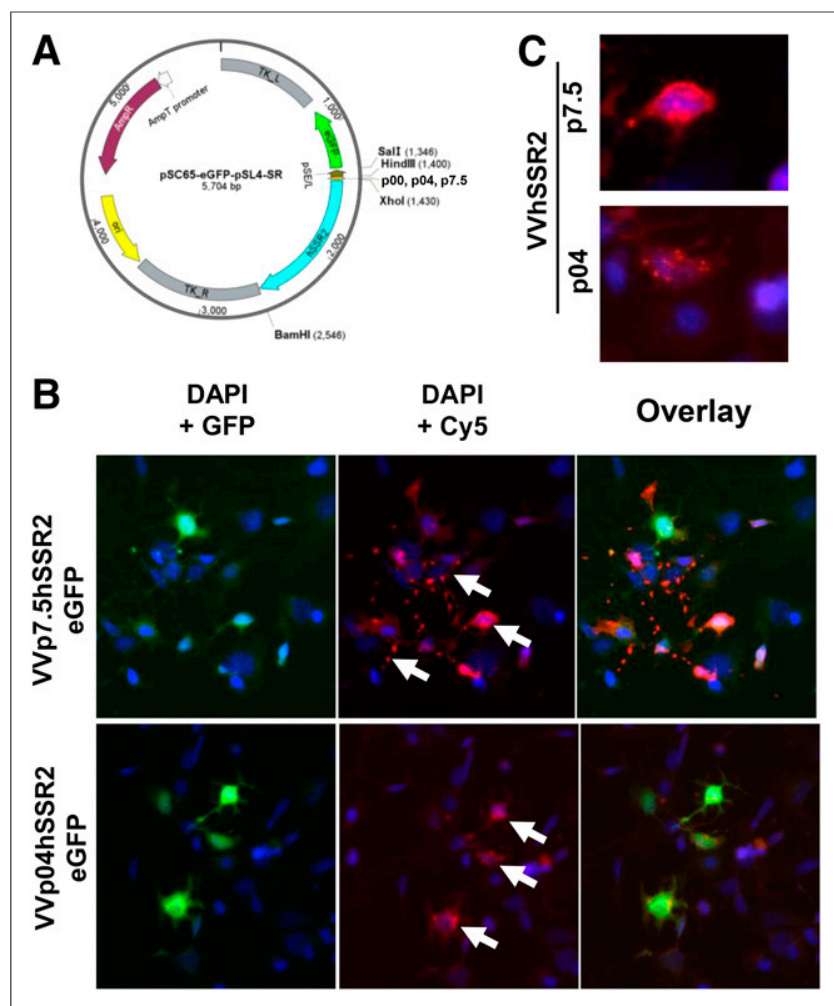

FIGURE 2. Late promoter for $h S S R 2$ vaccinia-based platform expression. (A) Diagram of vector used to generate recombinant VVhSSR2. (B) Immunofluorescence of U2OS cells infected by early/late p7.5 VV-hSRR2 and late p04. Arrows point to localization of hSSR2: more cytoplasmic homogenously distributed hSSR2 when using late promoter vs. compacted expression when $h S S R 2$ is under early/late promoter. Pink = Cy5; green = GFP from viral gene expression; blue = DAPI (4,6-diamidino-2-phenylindole). (C) Magnified view of specific cells with immunofluorescence staining. Cy5 = fluorophore conjugated to secondary antibody for hSSR2 detection.

by an in vivo imaging system (IVIS) (Fig. 3D). VV expression in tumors was confirmed after the sacrifice of animals by assessing the viral expression of eGFP directly by microscopy and by immunohistochemistry staining for vaccinia antigens (Fig. 3E). Interestingly, we also performed an immunohistochemistry staining to assess proliferating cells (Fig. 3E). We observed that regions showing vaccinia-infected cells resulted in dead tissue without any evidence of proliferating tumor cells. Confirming that these viruses may be controlling tumor growth, the parental $\mathrm{VV}$ and the $h N I S$-expressing derivatives were all able to significantly control the growth of the tumor and to increase the overall survival of the treated mice compared with the PBS control group (Figs. 3F and 3G).

\section{hNIS-Encoding Virus Can Be Imaged Using SPECT/CT}

To validate in vivo imaging using ${ }^{99} \mathrm{Tc}$, the tracer was injected $4 \mathrm{~d}$ after the intratumoral treatment of human HT29 xenografted tumors with PBS, parental VV, or $h N I S$-recombinant virus. After $1 \mathrm{~h}$ to allow the tracer to reach all organs, mice were imaged using small-animal SPECT/CT (Fig. 4). Consistent with earlier reports, the thyroid, salivary gland, stomach, bladder, and occasionally bowel were positive for ${ }^{99} \mathrm{Tc}$ signal (Fig. 4A) $(35,36)$. However, there were no radioactive signals for tumors treated with either PBS or nonexpressing hNIS virus (Fig. 4A). In contrast, mice treated with the hNIS-encoding oncolytic candidates showed strong positive ring-shaped signals in the tumor. Interestingly, we have noted that the signal persisted for at least $11 \mathrm{~d}$ after the initial viral injection (data not shown). Although these studies were initially performed with intratumoral delivery of virus, intravenous delivery of virus was also found to be sufficient for in vivo imaging of tumor using small-animal SPECT/CT (Fig. 4B), opening the possibility of using systemic virus delivery to image tumors at remote sites. With the aim to assess the safety profile of using a viral platform expressing NIS receptor, we determined the level of virus replication, hNIS expression, and radioactive

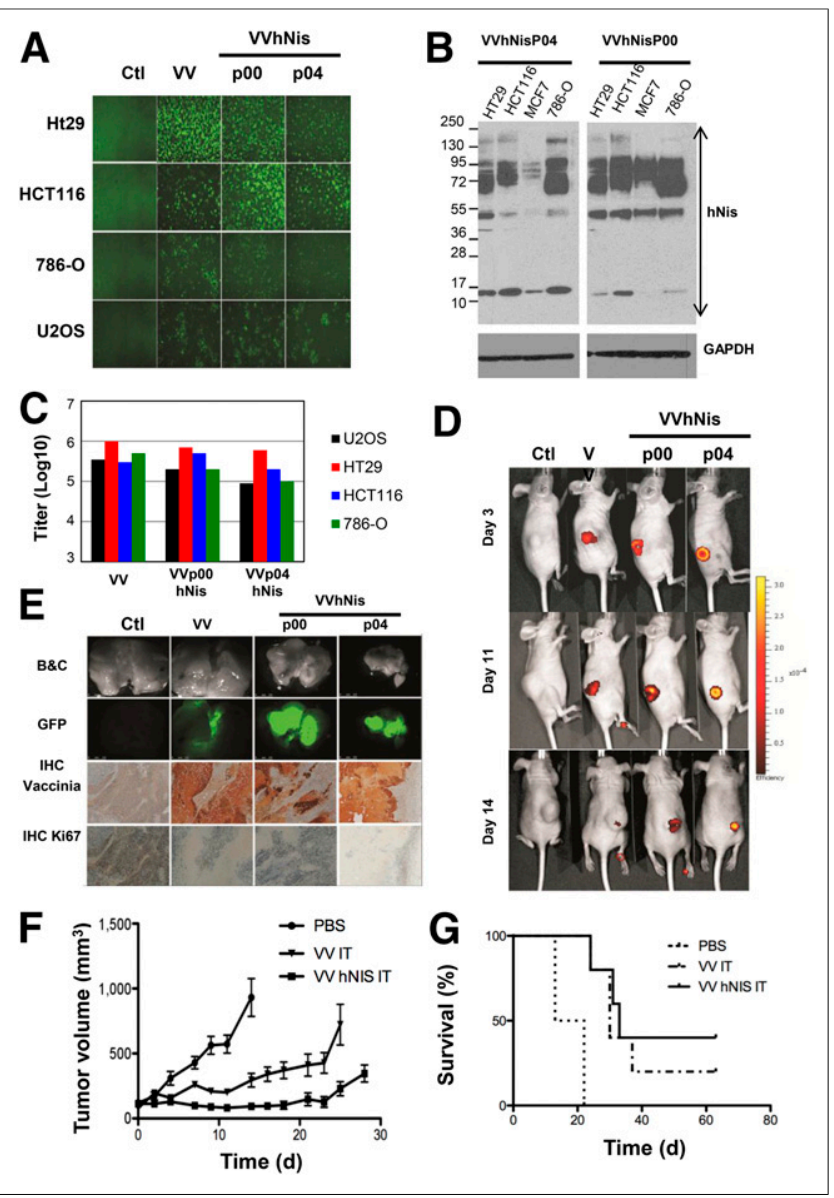

FIGURE 3. In vitro and in vivo infection of tumor cell with $\mathrm{hNIS}$-encoding viruses. (A) GFP images (48 h after infection) of HT29, HCT116, 786-O, and U2OS either uninfected (Ctl) or infected with VV or VV-hNIS p00 or p04 $(\mathrm{MOI}=0.01)$. (B) Western blot for NIS expression in tumor cells was performed as described earlier. (C) Viral titers (48 $\mathrm{h}$ after infection). (D) HT29 tumors were established in nude mice. Ten days later, mice were treated (intratumorally) with PBS (Ctl), VV, or VV-hNIS p00 or p04. GFP-associated virus expression was monitored using IVIS at 3, 11, and $14 \mathrm{~d}$ after virus administration. (E) Mice were sacrificed (day 14) and tumors harvested. Excised tumors were imaged for viral GFP expression, and paraffin-embedded blocks prepared from the tumors were screened by immunohistochemistry (IHC) for vaccinia antigens and Ki-67. (F) HT29 tumor volume was monitored for each group, and average tumor volumes are shown $(n=5)$. Treated groups vs. PBS are significantly different $(P<$ 0.001). Error bars are SEs. (G) HT29-bearing tumor mouse survival was monitored over time. Log-rank test indicates that treatment with $\mathrm{VV}$ or nonexpressing hNIS virus significantly extended survival compared with PBS $(P<0.01)$. B\&C = phase contrast; GAPDH = glyceraldehyde 3-phosphate dehydrogenase. 


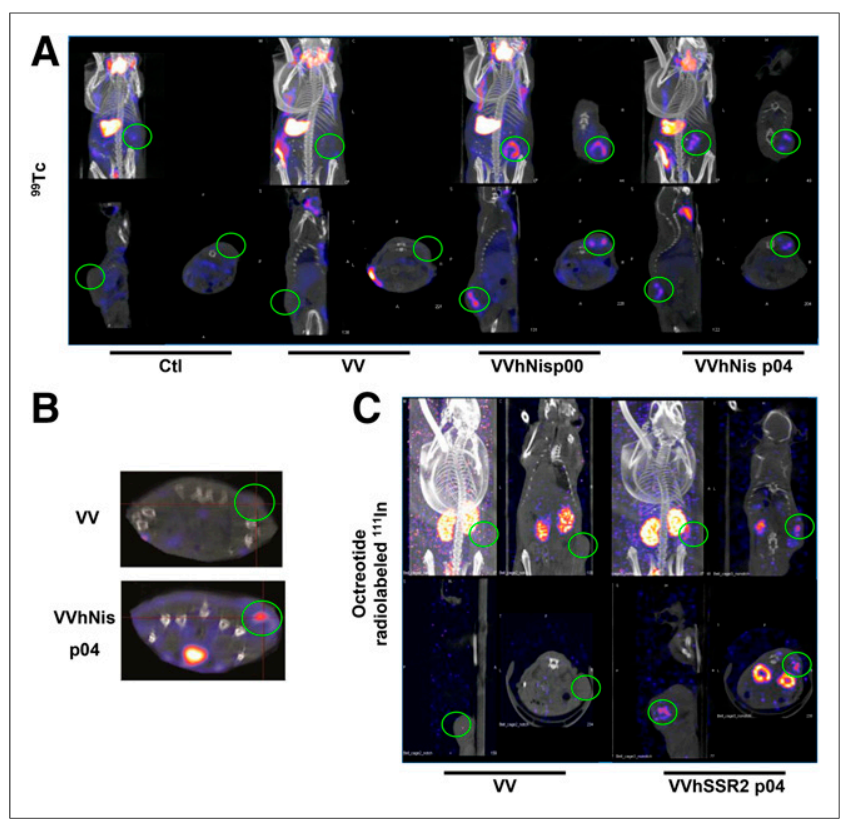

FIGURE 4. Radioisotope uptake for nude mice bearing HT29 tumors treated with control, hNIS, or hSSR2-encoding viruses. (A) HT29 tumors were established subcutaneously in mice before intratumoral injection with PBS (Ctl) or the indicated VV. Four days after virus treatment, mice were injected with ${ }^{99} \mathrm{Tc}$ radioisotope for small-animal SPECT/CT imaging. (B) Small-animal SPECT/CT images of HT29 tumor-bearing mice at day 4 after intravenous virus delivery. (C) HT29 tumors were injected intratumorally with VV or VV-hSSR2, and $4 \mathrm{~d}$ after virus treatment mice were injected with ${ }^{111} \mathrm{In}$-octreotide for small-animal SPECT/CT.

tracer accumulation in various tissues from tumor-bearing and tumor-free mice (Supplemental Fig. 1). At 24, 48, and $72 \mathrm{~h}$ after virus injection, ${ }^{99 \mathrm{~m} T c-p e r t e c h n e t a t e}$ was injected, and normal tissues (including blood, brain, bone, muscle, stomach, bladder, thyroid, ovaries) were harvested from both tumor-free and tumorbearing mice with the additional tumor tissue harvested from the latter group of mice. A $\gamma$-counter was then used to measure the radiation in each tissue (Supplemental Fig. 1A). Subsequently, portions of all tissues were homogenized and membrane proteins extracted for Western blotting to assay expression of the hNIS protein (Supplemental Fig. 1B). Finally, the remaining portions of the tissues were titered for VV (Supplemental Fig. 1C). As expected, we observed a strong radiation signal in tumor in addition to a strong signal in the few normal tissues in both tumor-free or tumor-bearing mice that are known to express endogenous murine NIS (thyroid, bladder, and stomach). Consistent with these findings, the virus was detected only in high amounts in tumor tissues (Supplemental Fig. 1C). This observation matched perfectly with the Western blotting, where expression of hNIS was seen only in the tumor tissue. In conclusion, based on tissue viral titres, significant amounts of virus replication occur primarily in the tumor tissue. This correlates well with our ability to detect the virusencoded hNIS protein only in tumor tissue and with the observation that the only tissues that accumulated the pertechnetate radiotracer, other than tissues known to express endogenous murine NIS, were the tumor tissues.

In parallel, the octreotide radiolabeled with ${ }^{111} \mathrm{In}$, a drug already in clinical use, was similarly effective for imaging tumor infected with virus candidates expressing hSSR2 (Fig. 4C). As expected, results mirrored those obtained with hNIS-expressing vaccinia, and tumor labeling with ${ }^{99} \mathrm{Tc}$ was observed, confirming the potential of this viral technology to be used for both imaging and antitumor therapeutics.

\section{hNIS-Encoding Virus Is Able to Infect and Localize Multiple Tumors by Imaging}

The murine tgMISIIR-Tag, a T-antigen-driven ovarian cancer model, makes several foci of tumor development in the ovaries and is an extremely difficult model to cure. We tested the vaccinia hNIS viral platform for imaging tumors in this model to assess the potential of this new therapeutic candidate (Fig. 5A). Interestingly, the systemic injection of the VV-hNIS virus revealed clear imaging of multiple tumor nodules in the tumor-bearing mice in vivo, using ${ }^{99} \mathrm{Tc}$. Replication of vaccinia in this model was evident by imaging of GFP expression in excised ovarian tumors (Fig. 5C). A survival study using the same ovarian tumor model (Fig. 5B) showed an enhanced survival of mice that received the hNIS virus with ${ }^{131} \mathrm{I}$ when compared with ${ }^{131} \mathrm{I}$ treatment alone. Together, these data strongly suggest the potential clinical use of viruses expressing symporter in combination with radioisotopes to localize and visualize tumor growth and the direct therapeutic potential of using viral platforms expressing symporter for treating various types of cancers.

Finally, using HT29 tumor, we examined the ability of VV-hNIS and VV-hSRR2 to replicate and also to accumulate injected radioisotopes in the tumor of a single animal (Fig. 6). Four days after injections of viruses, technetium and iodide radioisotopes were coinjected intraperitoneally $\left({ }^{111} \mathrm{In}\right)$ or intravenously $\left({ }^{99} \mathrm{Tc}\right)$ and tracers were followed by small-animal SPECT/CT. Results show that both radioisotopes accumulated in the tumor implantation, suggesting that hNIS- and hSRR2-encoding viruses can both be used simultaneously for imaging.

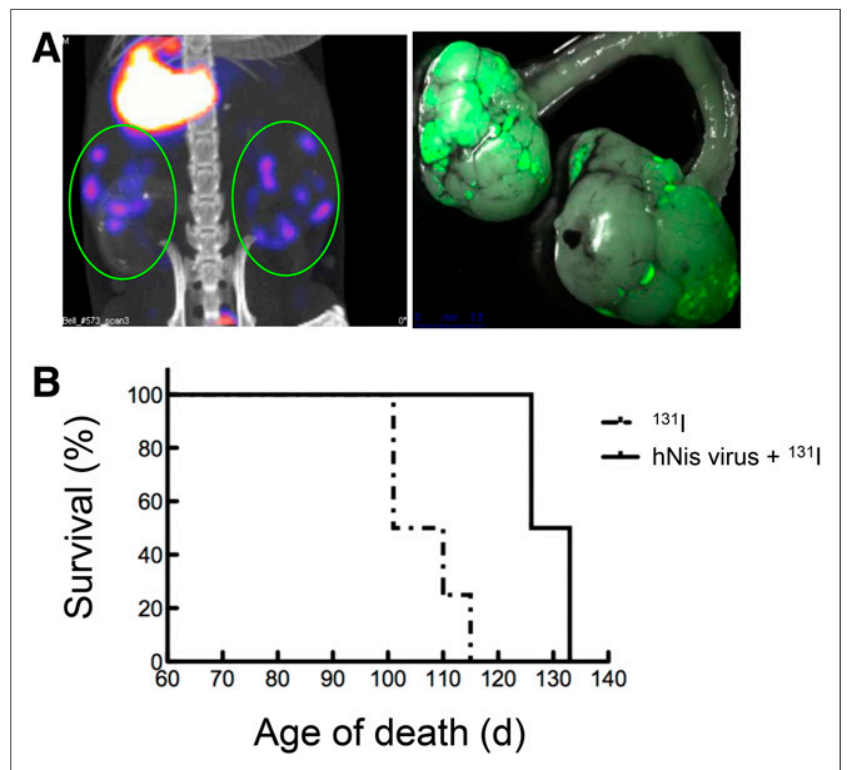

FIGURE 5. VV-hNIS is able to reveal and treat a transgenic ovarian tumor mouse model. (A) tgMISSIIR-TAg transgenic mice were treated with VVp04-hNIS. tgMISIIR-TAg mice, $90 \mathrm{~d}$ old, were injected intravenously with VV-hNIS, and $4 \mathrm{~d}$ later a small-animal SPECT/ CT image was obtained. (B) Survival of tgMISSIIR-Tag transgenic mice was monitored over time after treatment with VVp04-hNIS with 131I $(n=5)$. 


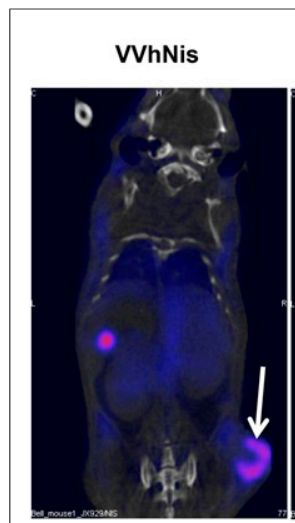

${ }^{99} \mathrm{Tc}$
VVhSSR2

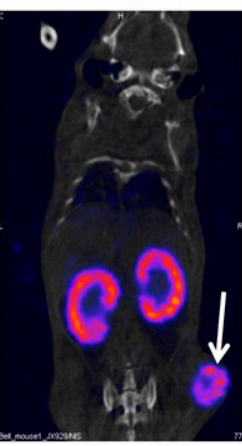

${ }^{111}$ In
VVhNis + VWhSSR2

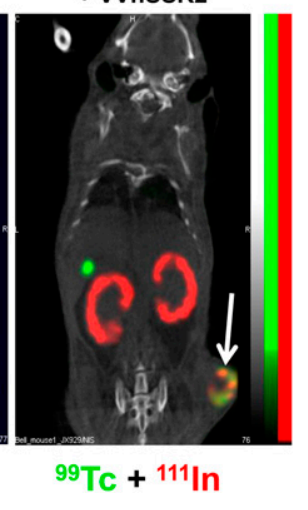

FIGURE 6. Dual VV-hNIS and VV-hSSR2 were used to visualize and treat tumors. HT29 tumors were injected with VV-hNIS and VV-hSSR2 intratumorally. Four days after virus treatment, mice were injected with ${ }^{99} \mathrm{Tc}$ and ${ }^{111} \mathrm{In}$ radioisotopes for small-animal SPECT/CT imaging.

\section{DISCUSSION}

In this study, we showed the potential use of VV expressing 2 different genes, NIS symporter and the SSR2 receptor in the aim of visualizing and treating tumors. Using a small-animal SPECT/ CT imaging system, we were able to clearly visualize and localize tumors in various mouse models. When this recombinant vaccinia expression platform was used, systemic administration of virus and subsequently radioisotopes led to accumulation of isotope in the tumor bed and improved survival in an ovarian cancer model.

Poxviruses continue to be important therapeutics for the prevention and treatment of human diseases. Vaccinia application for the eradication of smallpox has been one of the most important medical advances in human history, providing to the scientific and medical community strong data on the safety profile of the virus. A variety of poxvirus-based vaccine vectors have been developed for human infectious diseases and as agents for the treatment of cancer. This characteristic gives us the opportunity to combine 2 desirable effects: a cancer therapeutic and a cancer tool for tumor localization. The lytic ability of the platform has been described several times in various preclinical models but also in clinical trials. An important feature of this specific vaccinia viral platform is its strong ability to image the tumor after systemic injections, making this platform an attractive prospect for mapping and imaging tumors in patients with metastatic disease. Also, because of its large genome and its slow replication, as a viral platform, vaccinia can uniquely replicate in the tumor over the course of several days after systemic injection, giving the opportunity for recombinant gene to be expressed during a long period of time. We also show that the choice of vaccinia promoters regulating the expression of the transgenes is crucial. In fact, a late promoter, in both platforms expressing $h N I S$ or $h S S R 2$, is clearly more advantageous for maintained strong expression in tumor cells when compared with an early/late promoter. Moreover, we also observed that the SSR2 localization is optimal using late promoter. The radiotracer concentration taken up in tumor nodules is critical for capturing a strong enough signal for imaging using small-animal SPECT/CT and is obviously extremely dependent on the level of expression of the symporter/receptor. The strongest and most functional expression of the symporter and receptor in vivo was observed when we used late vaccinia promoter $\mathrm{p} 00$ or $\mathrm{p} 04$.

Importantly, we also conducted experiments to make a strong correlation between the tracer signal, hNIS symporter membrane expression, and viral replication. Vaccinia thymidine kinase knockout virus exhibited a strong safety profile based on its persistence only in tumors. hNIS protein expression was detected only in tumor as well, and accumulation of tracer correlated with NIS-positive tumor in addition to a few normal tissues known to express endogenous murine NIS (stomach, bladder, and thyroid notably). This viral dynamic suggests that VV has strong advantages in its use to carry and selectively express membrane proteins such as NIS. Furthermore, with virus titers in normal tissues that are more than 4 orders of magnitude lower than those found in tumors, we do not expect the virus to drive sufficient amounts of NIS protein expression to make a difference in the accumulation of the tracer.

We have chosen 2 different VV expression platforms to allow the imaging of tumor sites in vivo: one using hNIS and the second one SSR 2 transgene. These 2 genes operate differently and can offer complementary results when combined. hNIS has been shown to work well in the measles virus platform and is now in clinical evaluation. Using viruses as a gene therapy platform to target cancer cells and express NIS clearly shows promise in the treatment and visualization of tumors. Interestingly, accumulation of ${ }^{111}$ In radioisotopes will be only in live cells expressing NIS pump. Somatostatin receptor type 2 is a surface cell receptor that can be used as a target for specific targeted drugs. Notably, SSR2 is overexpressed in certain types of cancer. Using ${ }^{111}$ In-octreotide, a Food and Drug Administrationapproved drug, in patients with neuroendocrine tumors provides high-resolution images available to clinicians to help in diagnoses. The advantage of using this receptor is notably that SSR2 can be expressed on live and dead tumor cells, providing a different picture area of the tumor compared with using the hNIS gene viral platform.

The route of injection of therapeutic agent can be a source of great debate; VV-expressing specific reporter genes can help in the decision and contribute to a better success in the following clinical trials when used as a stand-alone or in combination with specific anticancer treatment such as checkpoint inhibitors.

\section{CONCLUSION}

Here, we report the use of 2 new vaccinia-based viruses expressing 2 different imaging genes for tumor visualization and treatment. Both platforms, with their respective radiolabeled counterparts, can be used independently or together, offering unique opportunities to diagnose suspected cancer, visualize multiple metastasis with high-quality small-animal SPECT/CT imaging, and provide a treatment through the use of the oncolytic viral platform activating multiple mechanisms of action against tumors.

\section{DISCLOSURE}

This work was supported by grants from the National Cancer Institute of Canada, Terry Fox Foundation, Natural Sciences and Engineering Research Council of Canada, and the Canadian Institutes of Health Research to John C. Bell and R. Glenn Wells. No other potential conflict of interest relevant to this article was reported. 


\section{REFERENCES}

1. Bell JC. Interfering with tumor pathways that augment viral oncolysis. Mol Ther. 2011;19:2108-2109.

2. Bell JC, McFadden G. Editorial overview: oncolytic viruses-replicating virus therapeutics for the treatment of cancer. Curr Opin Virol. 2015;13: viii-ix.

3. Auer R, Bell JC. Oncolytic viruses: smart therapeutics for smart cancers. Future Oncol. 2012;8:1-4.

4. Russell SJ, Peng KW, Bell JC. Oncolytic virotherapy. Nat Biotechnol. 2012;30: 658-670.

5. Stojdl DF, Lichty BD, tenOever BR, et al. VSV strains with defects in their ability to shutdown innate immunity are potent systemic anti-cancer agents. Cancer Cell. 2003;4:263-275.

6. Rintoul JL, Wang J, Gammon DB, et al. A selectable and excisable marker system for the rapid creation of recombinant poxviruses. PLoS One. 2011;6: e24643.

7. Mastrangelo MJ, Maguire $\mathrm{HC}$ Jr, Eisenlohr LC, et al. Intratumoral recombinant GM-CSF-encoding virus as gene therapy in patients with cutaneous melanoma. Cancer Gene Ther. 1999;6:409-422.

8. Erbs P, Findeli A, Kintz J, et al. Modified vaccinia virus Ankara as a vector for suicide gene therapy. Cancer Gene Ther. 2008;15:18-28.

9. Smith GL, Mackett M, Moss B. Infectious vaccinia virus recombinants that express hepatitis B virus surface antigen. Nature. 1983;302:490-495.

10. Smith GL, Murphy BR, Moss B. Construction and characterization of an infectious vaccinia virus recombinant that expresses the influenza hemagglutinin gene and induces resistance to influenza virus infection in hamsters. Proc Natl Acad Sci USA. 1983;80:7155-7159.

11. Cadoz M, Strady A, Meignier B, et al. Immunisation with canarypox virus expressing rabies glycoprotein. Lancet. 1992;339:1429-1432.

12. Kantoff PW, Schuetz TJ, Blumenstein BA, et al. Overall survival analysis of a phase II randomized controlled trial of a poxviral-based PSA-targeted immunotherapy in metastatic castration-resistant prostate cancer. J Clin Oncol. 2010;28:1099-1105.

13. Harrop R, Drury N, Shingler W, et al. Vaccination of colorectal cancer patients with modified vaccinia ankara encoding the tumor antigen 5T4 (TroVax) given alongside chemotherapy induces potent immune responses. Clin Cancer Res. 2007; 13:4487-4494.

14. Dasgupta S, Bhattacharya-Chatterjee M, O'Malley BW Jr, Chatterjee SK. Recombinant vaccinia virus expressing interleukin-2 invokes anti-tumor cellular immunity in an orthotopic murine model of head and neck squamous cell carcinoma. Mol Ther. 2006;13:183-193.

15. Lorenz MG, Kantor JA, Schlom J, Hodge JW. Anti-tumor immunity elicited by a recombinant vaccinia virus expressing CD70 (CD27L). Hum Gene Ther. 1999;10: 1095-1103.

16. Lorenz MG, Kantor JA, Schlom J, Hodge JW. Induction of anti-tumor immunity elicited by tumor cells expressing a murine LFA-3 analog via a recombinant vaccinia virus. Hum Gene Ther. 1999;10:623-631.

17. Evgin L, Vaha-Koskela M, Rintoul J, et al. Potent oncolytic activity of raccoonpox virus in the absence of natural pathogenicity. Mol Ther. 2010;18:896-902.

18. Kanesa-thasan N, Smucny JJ, Hoke CH, et al. Safety and immunogenicity of NYVAC-JEV and ALVAC-JEV attenuated recombinant Japanese encephalitis virus: poxvirus vaccines in vaccinia-nonimmune and vaccinia-immune humans. Vaccine. 2000;19:483-491.
19. Bejon P, Ogada E, Mwangi T, et al. Extended follow-up following a phase $2 \mathrm{~b}$ randomized trial of the candidate malaria vaccines FP9 ME-TRAP and MVA ME-TRAP among children in Kenya. PLoS One. 2007;2:e707.

20. Huygen K, Content J, Denis O, et al. Immunogenicity and protective efficacy of a tuberculosis DNA vaccine. Nat Med. 1996;2:893-898.

21. Parato KA, Breitbach CJ, Le Boeuf F, et al. The oncolytic poxvirus JX-594 selectively replicates in and destroys cancer cells driven by genetic pathways commonly activated in cancers. Mol Ther. 2012;20:749-758.

22. McCart JA, Ward JM, Lee J, et al. Systemic cancer therapy with a tumor-selective vaccinia virus mutant lacking thymidine kinase and vaccinia growth factor genes. Cancer Res. 2001;61:8751-8757.

23. Le Boeuf F, Diallo JS, McCart JA, et al. Synergistic interaction between oncolytic viruses augments tumor killing. Mol Ther. 2010;18:888-895.

24. Micali S, Bulotta S, Puppin C, et al. Sodium iodide symporter (NIS) in extrathyroidal malignancies: focus on breast and urological cancer. $B M C$ Cancer. 2014;14:303.

25. Appetecchia M, Baldelli R. Somatostatin analogues in the treatment of gastroenteropancreatic neuroendocrine tumours, current aspects and new perspectives. J Exp Clin Cancer Res. 2010;29:19.

26. De La Vieja A, Dohan O, Levy O, Carrasco N. Molecular analysis of the sodium/ iodide symporter: impact on thyroid and extrathyroid pathophysiology. Physiol Rev. 2000;80:1083-1105.

27. Riesco-Eizaguirre G, Santisteban P. A perspective view of sodium iodide symporter research and its clinical implications. Eur J Endocrinol. 2006;155:495-512.

28. Galanis E, Atherton PJ, Maurer MJ, et al. Oncolytic measles virus expressing the sodium iodide symporter to treat drug-resistant ovarian cancer. Cancer Res. 2015;75: 22-30.

29. Liu Y, Lu D, Zhang Y, Li S, Liu X, Lin H. The evolution of somatostatin in vertebrates. Gene. 2010;463:21-28.

30. Grimberg A. Somatostatin and cancer: applying endocrinology to oncology. Cancer Biol Ther. 2004;3:731-733.

31. Garson K, Gamwell LF, Pitre EM, Vanderhyden BC. Technical challenges and limitations of current mouse models of ovarian cancer. J Ovarian Res. 2012;5:39.

32. Peyrottes I, Navarro V, Ondo-Mendez A, et al. Immunoanalysis indicates that the sodium iodide symporter is not overexpressed in intracellular compartments in thyroid and breast cancers. Eur J Endocrinol. 2009;160:215-225.

33. Huc-Brandt S, Marcellin D, Graslin F, et al. Characterisation of the purified human sodium/iodide symporter reveals that the protein is mainly present in a dimeric form and permits the detailed study of a native C-terminal fragment. Biochim Biophys Acta. 2011;1808:65-77.

34. Castro MR, Bergert ER, Beito TG, McIver B, Goellner JR, Morris JC. Development of monoclonal antibodies against the human sodium iodide symporter: immunohistochemical characterization of this protein in thyroid cells. J Clin Endocrinol Metab. 1999;84:2957-2962.

35. Wapnir IL, Goris M, Yudd A, et al. The $\mathrm{Na}+/ \mathrm{I}-$ symporter mediates iodide uptake in breast cancer metastases and can be selectively down-regulated in the thyroid. Clin Cancer Res. 2004;10:4294-4302.

36. Harun-Or-Rashid M, Asai M, Sun XY, Hayashi Y, Sakamoto J, Murata Y. Effect of thyroid statuses on sodium/iodide symporter (NIS) gene expression in the extrathyroidal tissues in mice. Thyroid Res. 2010;3:3. 